\title{
Survey on Convex Drawing of Planar Graph
}

\author{
Sharifa Rania Mahmud \\ Department of Computer Science and Engineering, Military Institute of Science and Technology, Dhaka, \\ Bangladesh
}

\begin{abstract}
This paper presented study on convex drawing of planar graph. In graph theory, a planar graph is a graph that can be embedded in the plane. A planar graph is one that can be drawn on a plane in such a way that there are no "edge crossings," i.e. edges intersect only at their common vertices. Convex polygon has all interior angles less than or equal to $180^{\circ}$. A graph is called a convex drawing if every facial cycle (face) is drawn as a convex polygon. In a convex drawing of a planar graph, all edges are drawn by straight line segments in such a way that every face boundary in a convex polygon. This paper describes some of the recent works on convex drawing on planar graph.
\end{abstract}

Keywords - Biconnected, Convex Drawing, Internally Triconnected, Planar Graph.

\section{Introduction}

The problem of drawing a planar graph often arises in design automation for electrical networks, particularly VLSI circuits, software engineering and bioinformatics. Now, the main interest is to draw a planar graph so that one can easily and rapidly recognize its structure such as the adjacency of vertices and the connectivity property. A graph $G=(V, E)$ is called planar if its vertices and edges are drawn as points and curves in the plane so that no two curves intersect except at their endpoints, where no two vertices are drawn at the same point. In such a drawing, the plane is divided into several connected regions, each of which is called a face. A face is characterized by the cycle of $G$ that surrounds the region. Such a cycle is called a facial cycle. A set $F$ of facial cycles in a drawing is called an embedding of a planar graph $G$. A plane graph $G=(V, E, F)$ is a planar graph $G=(V, E)$ with a fixed embedding $F$ of $G$, where we always denote the outer facial cycle in $F$ by $f_{o}$ $\in F$. One of the feasible methods for the purpose is a convex drawing in which all the edges are drawn by straight line segments without any crossing so that all the face boundaries are convex polygons.

\section{Literature Review}

Note that not all planar graphs admit a convex drawing. Tutte [1] gave a necessary and sufficient condition for a triconnected plane graph to admit a convex drawing. He also showed that every triconnected plane graph with a given boundary drawn as a convex polygon admits a convex drawing using the polygonal boundary. That is, when the vertices on the boundary are placed on a convex polygon, inner vertices can be placed on suitable positions so that each inner facial cycle forms a convex polygon. More specifically, he proposed a "barycentric mapping" method which computes a convex drawing of a triconnected plane graph with $n$ vertices by solving a system of $O(n)$ linear equations and so usually requires $O\left(n^{3}\right)$ computation time [2]. Hong and Nagamochi [13] proved that every internally triconnected hierarchical plane graph with the outer facial cycle drawn as a convex polygon admits a convex drawing.

Later, Thomassen [3] gave a necessary and sufficient condition for a biconnected plane graph to admit a convex drawing. Based on this result, Chiba et al. [4] presented a linear time algorithm for finding a convex drawing (if any) for a biconnected plane graph with a specified convex boundary. In general, the convex drawing problem has been well investigated for the last ten years by the Graph Drawing community. For example, a problem of convex drawing of graphs with grid constraints has been well studied $[5,6,7,8]$. A convex drawing is called a convex grid drawing if all the vertices are restricted to be placed on grid points. Every triconnected plane graph has a convex grid drawing on an $(n-2) \times(n-2)$ grid, and such a grid drawing can be found in linear time [7]. A linear time algorithm for finding a convex grid drawing of four-connected plane graphs with four or more vertices on the outer face was presented in [8]. Another variation of convex drawing with minimum outer apices was introduced in [9]. For constructing a strictly convex drawing of graphs, see [10].

Recently in 2010, introduce a new problem [11] of drawing planar graphs with non-convex boundary constraints. A straight-line drawing is called an inner-convex drawing if every inner facial cycle is drawn as a convex polygon. It is proved that every triconnected plane graph admits an inner-convex drawing if its boundary is fixed with a star-shaped polygon $P$, i.e., a polygon $P$ whose kernel (the set of all points from which all points in $P$ are visible) is not empty [11]. Note that this is an extension of the classical result by Tutte [1] since any convex polygon is a star-shaped polygon. The paper also presented a linear time algorithm for computing an 
inner-convex drawing of a triconnected plane graph with a star-shaped boundary [11]. Battista et al. [12] also present Non-convex representations of graphs in 2009. SH Hong and H Nagamochi present [14] a linear-time algorithm for symmetric convex drawings of internally triconnected Plane Graphs in 2010.

\section{Prilimineries}

A vertex in a connected graph is called a cut vertex if its removal from $G$ results in a disconnected graph. A connected graph is called biconnected if it is simple and has no cut vertex. Similarly, a pair of vertices in a connected graph is called a cut pair (or separation pair) if its removal from $G$ results in a disconnected graph. A connected graph is called triconnected if it is simple and has no cut pair. We say that a cut pair $\{u, v\}$ separates two vertices $s$ and $t$ if $s$ and $t$ belong to different components in $G-\{u, v\}$. A graph $G=(V, E)$ is called planar if its vertices and edges are drawn as points and curves in the plane so that no two curves intersect except at their endpoints, where no two vertices are drawn at the same point. In such a drawing, the plane is divided into several connected regions, each of which is called a face. A face is characterized by the cycle of $G$ that surrounds the region. Such a cycle is called a facial cycle. A set $F$ of facial cycles in a drawing is called an embedding of a planar graph $G$. A plane graph $G=(V, E, F)$ is a planar graph $G=(V, E)$ with a fixed embedding $F$ of $G$, where we always denote the outer facial cycle in $F$ by $f_{o} \in F$. A vertex (respectively, an edge) in $f_{o}$ is called an outer vertex (respectively, an outer edge), while a vertex (respectively, an edge) not in $f_{o}$ is called an inner vertex (respectively, an inner edge). A path $Q$ between two vertices $s$ and $t$ in $G$ is called inner if every vertex in $V(Q)-\{s, t\}$ is an inner vertex. The region enclosed by a facial cycle $f \in F$ may be denoted by $f$ for simplicity. A biconnected plane graph $G$ is called internally triconnected if, for any cut pair $\{u, v\}, u$ and $v$ are outer vertices and each component in $G-\{u, v\}$ contains an outer vertex. Note that every inner vertex in an internally triconnected plane graph must be of degree at least 3 . For a cut pair $\{u, v\}$ of an internally triconnected plane graph $G=(V, E, F)$, if $u$ and $v$ are not adjacent and there is an inner facial cycle $f \in F$ such that $\{u, v\} \in V$ $(f)$, we say that $f$ separates two vertices $s$ and $t$ if the cut pair $\{u, v\}$ separates them.

\subsection{Linear Algorithms for Convex Drawings of Planar Graphs}

\section{Description of Some Recent Works}

Chiba et al. [4] presented a linear time algorithm for finding a convex drawing (if any) for a biconnected plane graph with a specified convex boundary in 1984. In the paper authors present two linear algorithms for the convex drawing problem of planar graphs, drawing and testing algorithms. The former draws a given planar graph $G$ convex if possible: it extends a given convex polygon of an outer facial cycle of $G$ into a convex drawing of $G$. The latter tests the possibility; it determines whether $G$ has an outer facial cycle extendable to a convex drawing of $G$. These two algorithms together can efficiently yield various convex drawings of a planar graph. Both the time and space of these algorithms are linear, so optimal to within a constant factor.

Thomassen [3] gave a short proof to a strong version of the Tutte's result [1]. The drawing algorithm of this paper is based on it. On the other hand the author's modify their results into a form suitable for the convex testing, which is represented in terms of 3-connected or triconnected components. Using the form, they show that convex testing of a graph $G$ can be reduced to the planarity testing of a certain graph obtained from $G$.

Lemma 1 (Thomassen [3]) Let $G$ be a 2-connected plane graph with the outer facial cycle $S$, and let $S^{*}$ be a convex polygon of $S$. Let $P_{1}, P_{2}, \ldots, P_{K}$ be the paths in $S$, each corresponding to a side of $S^{*}$ (Thus $S^{*}$ is a $k$-gon. It should be noted that not every vertex of the cycle $S$ is an apex of the polygon $\left.S^{*}\right)$. Then $S^{*}$ is extendable if and only if Condition I below holds.

\section{Condition I}

(a) For each vertex $v$ of $G-V(S)$ having degree at least three in $G$, there exist three paths disjoint expect $v$, each joining $v$ and a vertex of $S$.

(b) $\quad G-V(S)$ has no connected component $C$ such that all the vertices on $S$ adjacent to vertices in $C$ lie on a single path $P_{i}$ and no two vertices in each $P_{i}$ are joined by an edge not in $S$.

(c) Any cycle of $G$ which has no edge in common with $S$ has at least three of degree $\geq 3$ in $G$.

\subsubsection{Convex Drawing Algorithm}

Let $G$ be a given 2-connected plane graph with the outer facial cycle $S$, and let $S^{*}$ be an extendable convex polygon of $S$. For simplicity we reduce the drawing of the graph $G$ into that of a graph $G$ ' which has no vertex of degree two not on $S$. 
Step 1: For each vertex $v$ of degree two not on $S$, replace $v$ together with the two edges incident to $v$ by a single edge joining the vertices adjacent to $v$. Let $G$ ' be the resulting graph.

Step 2: Call the procedure DRAW $\left(G^{\prime}, S, S^{*}\right)$ below to extend $S^{*}$ into a convex drawing of $G^{\prime}$.

Step 3: For each deleted vertex of degree 2 determine its position on the straight line segment joining the two vertices adjacent to the vertex.

Here DRAW $\left(G, S, S^{*}\right)$ procedure extends a convex polygon $S^{*}$ of the outer facial cycle $S$ of a plane graph $G$ into a convex drawing of $G$, where $G$ has no vertex of degree 2 not on $S$.

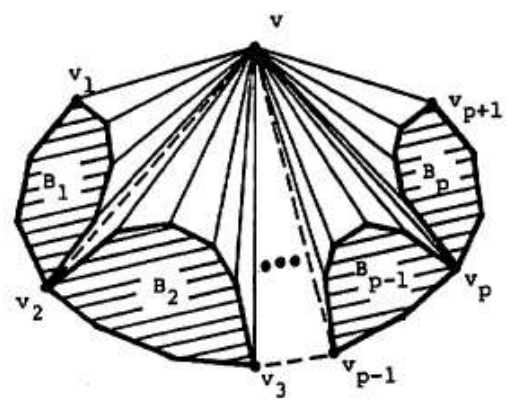

Figure 1. Reduction of the convex drawing of $G$ into subproblems.

Theorem 1 Let $G$ be a 2-connected plane graph with the outer facial cycle $S$ and let $S^{*}$ be an extendable convex polygon of $S$. Then the algorithm CONVEX-DRAWING extends $S^{*}$ into a convex drawing of $G$ and uses linear time and space.

\subsubsection{Testing Algorithm}

Modify Condition I in Lemma 1 into a form suitable for our purpose. One may easily notice that the existence of a convex drawing of a graph $\mathrm{G}$ heavily depends on the structure of 3-connected components of G.

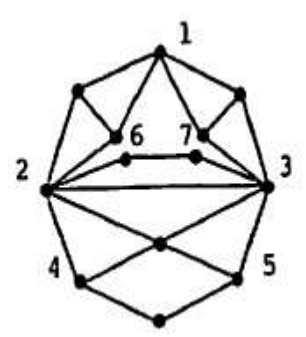

(a)

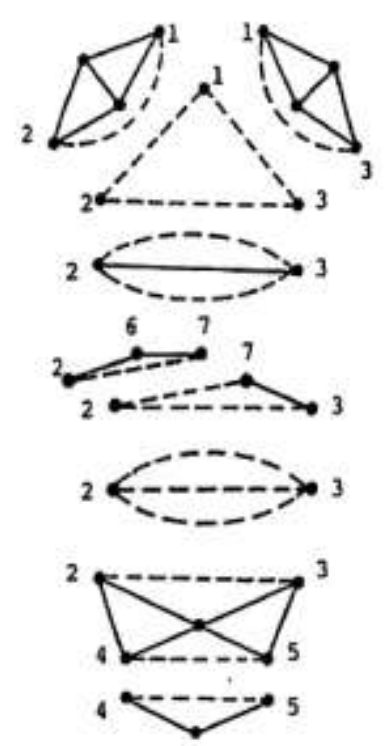

(b)

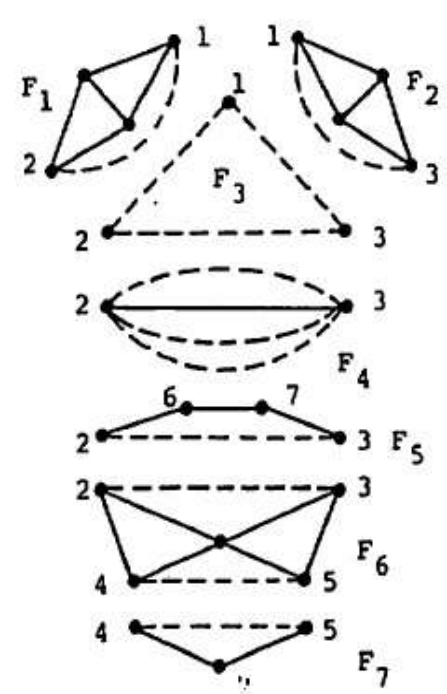

(c)
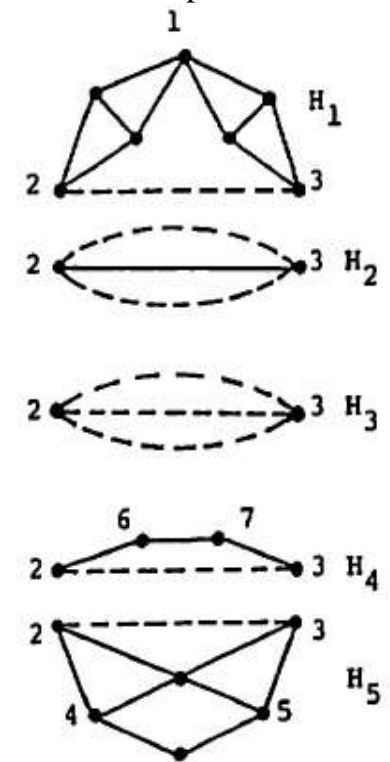

(d)

Figure 2. Decomposition of a graph, where virtual edges are written by dashed lines (a) A 2-connected graph $G$, (b) split component of $G$, (c) 3-connected of $G$, (d) $\{2,3\}$-split components of $G$ with one exceptions $H_{3}$.

Lemma 2 Let $G=(V, E)$ be a 2-connected plane graph with the outer facial cycles $S$ and let $S^{*}$ be e strict convex polygon of $S$. Then $S^{*}$ is extendable if and only if $g$ and $S$ satisfies the following Condition II.

\section{Condition II}

(a) G has no forbidden separation pair.

(b) For each critical separation pair $\{x, y\}$ of $G$ there exists at most one $\{x, y\}$-split component having no edge of S. Moreover such an $\{x, y\}$-split component is either a bond if $(x, y) \in E$ or a ring otherwise.

\section{Testing Algorithm}

The testing of Condition II can be reduced to the planarity testing of a certain graph. 
Lemma 3 Suppose that 2-connected planar graph G has no forbidden separation pair and has exactly one critical separation pair. Then $G$ has a convex drawing.
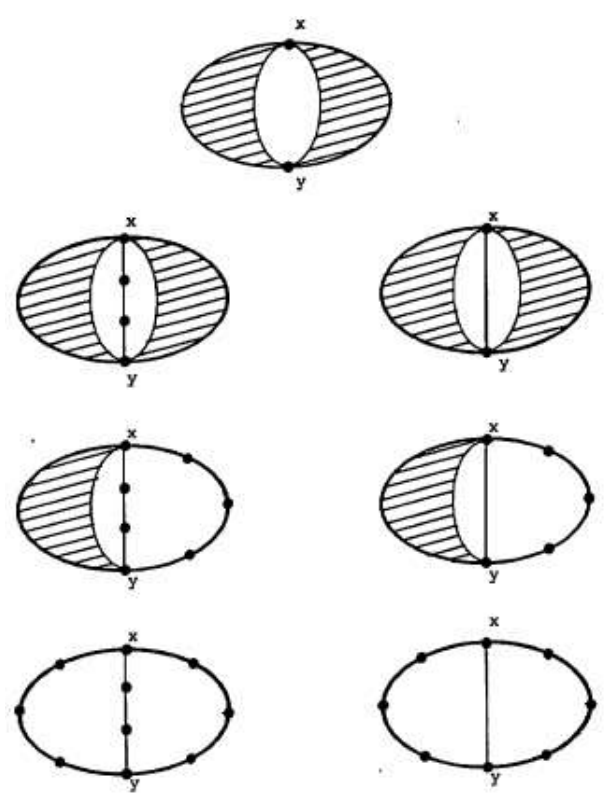

Figure 3. Seven types of plane graph having exactly one critical separation pair $\{\mathrm{x}, \mathrm{y}\}$.

Theorem 2 Suppose that a 2-connected planar graph $G$ has no forbidden separation pair and has two or more critical separation pairs. Apply the following operation to $G$ for every critical separation pair $\{x, y\}$ of $G$, if $(x$, y) $\epsilon E$, then delete edge $(x, y)$ from $G$, otherwise and if exactly one $\{x, y\}$-split component is a ring, and then delete the $x-y$ path in the component from $G$. Let $G_{1}$ be the resulting graph. (Graph $G$, and Gl are illustrated in Fig. 4(a) and 4(b) respectively). Then $S$ is an extendable facial cycle of $G$ if and only if $S$ is a facial cycle of $G_{I}$ which contains all the vertices of critical separation pairs of $G$.

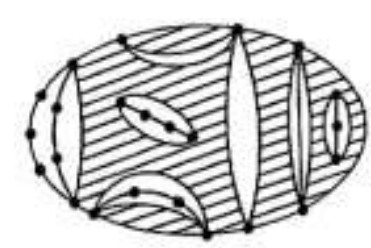

(a)

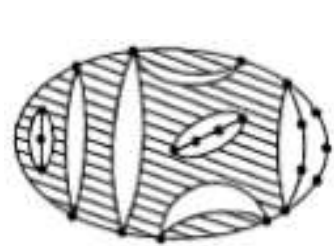

(b)

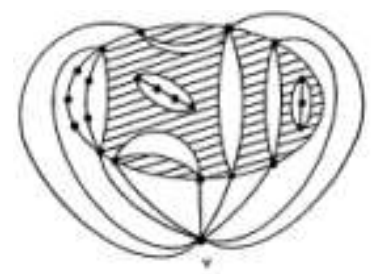

(c)

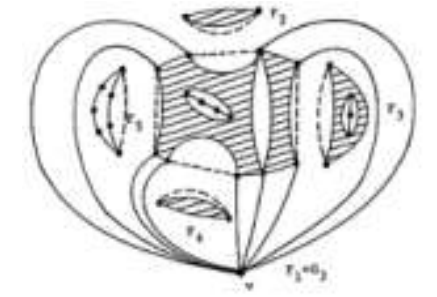

(d)

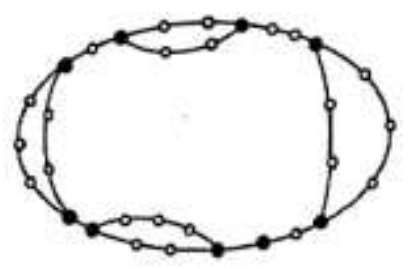

(e)

Figure 4. Illustration of Graphs, (a) $G$, (b) $G_{1}$, (c) $G_{2}$, (d) $F_{1}=G_{3}, F_{2}, F_{3}$ and $F_{4}$ are in group, (b) and $F_{5}$ are in group (c) and; (e) the union of all the extendable facial cycles of $G$.

Then it has to test convex testing by CONVEX-TESTING algorithm. The algorithm finds an extendable facial cycle $S$ if any. Then the authors show how to generate all the extendable facial cycles $G$. If the given graph $G$ has exactly one critical separation pair, then one can easily enumerate all the extendable facial cycles, since $G$ has at most four such facial cycles, as verified by checking the seven types in Fig. 3.

\subsubsection{Example}

The algorithms CONVEX-TESTING and CONVEX-DRAWING have been implemented in PASCAL and run on a small computer FACOM 230/38s. Experiments indicate that the algorithm can find all the extendable facial cycles of a graph having 150 edges in less than 6 seconds. The authors illustrate a 
computational example in Fig. 6. Fig. 6(a) depicts an input graph $\mathrm{G}$ having 50 vertices and 83 edges. Algorithm CONVEX-TESTING finds all the extendable facial cycles of $G$, the union of which is represented as shown in Fig. 5(b). A pair of paths, either of which can be a part of an extendable facial cycle, are in parentheses. Since there are two pairs of such paths, Fig. 5(b) represents the four extendable facial cycles:

$\mathrm{C}_{1}=15-16-1-2-3-4-5-6-7-8-9-10-11-12-13-14$

$\mathrm{C}_{2}=15-16-1-2-3-4-5-6-7-8-9-10-11-49-48-47$

$\mathrm{C}_{3}=15-16-1-2-3-31-32-7-8-9-10-11-12-13-14$

$\mathrm{C}_{4}=15-16-1-2-3-31-32-7-8-9-10-11-49-48-47$

The cycle $C_{l}$ is indicated by bold lines in Fig. 5(a). When both a plane graph $G$ with the outer cycle $C_{l}$ and convex polygon (regular 16-gon) of $C_{l}$ are given, algorithm CONVEX-DRAWING obtains a convex drawing of $G$, which is depicted in Fig. 5(c).

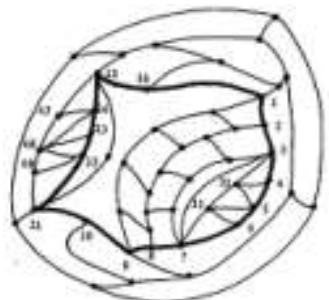

(a)
THE EXTENDABLE FACLAL CYCIES OF GIVEN GRAPH $1516123(456)(3132) 7891011(121314)(494847)$

(b)

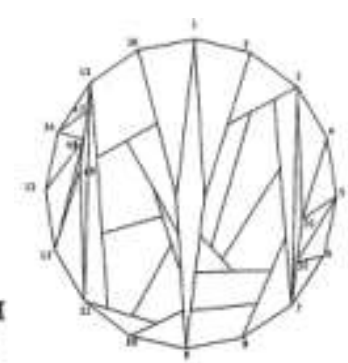

(c)

Figure 5. A computational example (a) Input graph G (some vertex numbers are not shown for simplicity), (b) Output of CONVEX-TESTING, (c) Output of CONVEX-DRAWING.

\subsection{Convex Drawing of Planar Graphs with Non-convex Boundary Constraints}

Hong and Nagamochi study a new problem [11] of convex drawing of planar graphs with non-convex boundary constraints. It is proved that every triconnected plane graph whose boundary is fixed with a starshaped polygon admits a drawing in which every inner facial cycle is drawn as a convex polygon. This is an extension of the classical result by Tutte [1] since any convex polygon is a star-shaped polygon. They also prove that every four-connected plane graph whose boundary is fixed with a crown-shaped polygon admits such a drawing, called an inner-convex drawing. They present an algorithm to construct an inner-convex drawing in linear time.

One can easily observe that not every triconnected plane graph has a convex drawing if its boundary is fixed as a non-convex polygon. For example, Fig. 6 shows three examples of plane graphs which have no convex drawing; the inner facial cycle $f_{1}$ in Fig. 6(b) (respectively, one of the inner facial cycles $f_{1}$ and $f_{2}$ in Figs. 6(a) and (c)) cannot be drawn as a convex polygon.

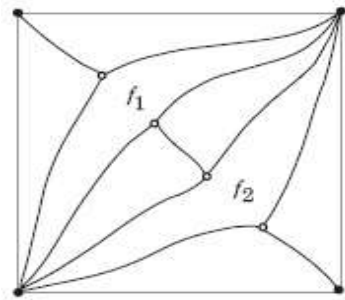

(a)

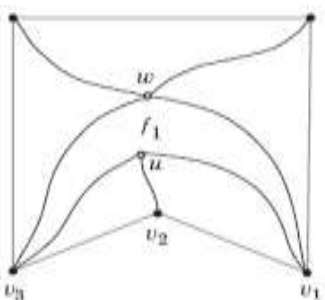

(b)

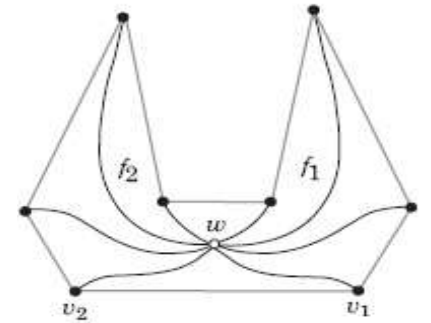

(c)

Figure 6. (a) A biconnected plane graph with a convex boundary; (b) An internally triconnected plane graph with a star-shaped boundary; (c) A triconnected plane graph whose boundary is crown-shaped but not starshaped.

Lemma 4 Every triconnected plane graph $G=(V, E, F)$ has a spanning tree T such that each vertex $v \in V\left(f_{o}\right)$ is a leaf of $T$. Such a tree can be found in linear time.

Lemma 5 Let $G=(V, E, F)$ be a 4-connected plane graph, and denote the vertices in $f_{o}$ by $v_{1}, v_{2}, \ldots, v_{q}$ such that they appear in this order when we walk along $f_{o}$ in the clockwise order (see Fig. 7(a)). Let $Q$ be a subpath of the outer facial cycle $f_{o}$ with $|V(Q)| \geq 2$, where the vertices $V(Q)$ are denoted by $v_{g+1}, v_{g+2}, \ldots, v_{q}$. Let $v_{g+1}^{\prime}$ denote the first inner vertex when we visit all neighbors of $v_{g+1}$ in the clockwise order starting with $v_{g+2}$, and $v^{\prime} q$ be the first inner vertex when we visit all neighbours of $v_{q}$ in the anticlockwise order starting with $v_{q-1}$. Then, 
(i) $\quad G-V\left(f_{o}\right)$ and $G^{\prime}=G-\left(V\left(f_{o}\right)-V(Q)\right)$ are both internally triconnected.

(ii) Let $B$ be the set of vertices in the boundary of $G$ '. Then, there is a matching $M=\left\{\left(v_{j}, w_{j}\right) \in E \mid j=1, \ldots\right.$, $g\} \cup\left\{\left(v_{g+1}, v_{g+1}^{\prime}\right),\left(v_{q}, v_{q}^{\prime}\right)\right\}$ such that $w_{j} \in B, j=1, \ldots, g$ (see Fig. $\left.7(b)\right)$. Such a matching $M$ can be obtained in linear time.

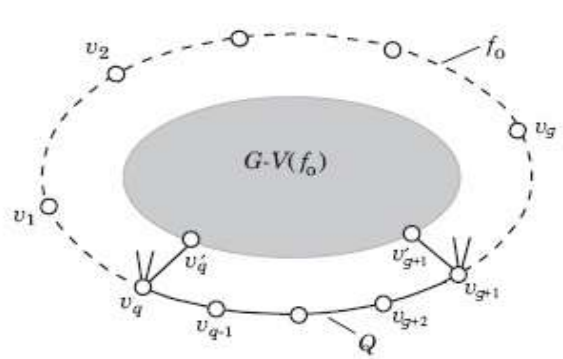

(a)

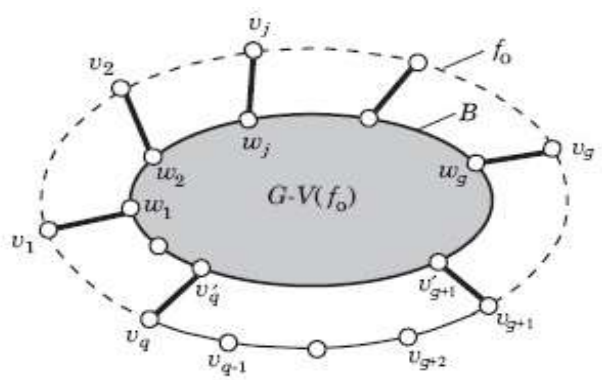

(b)

Figure 7. (a) Subgraph $G^{-} V\left(f_{o}\right)$ and subpath $Q$ in $f_{o}$; (b) Matching $M$ joining outer vertices and inner vertices, where matching edges are displayed by thick lines.

\subsubsection{Arch free Paths and Arch free Trees}

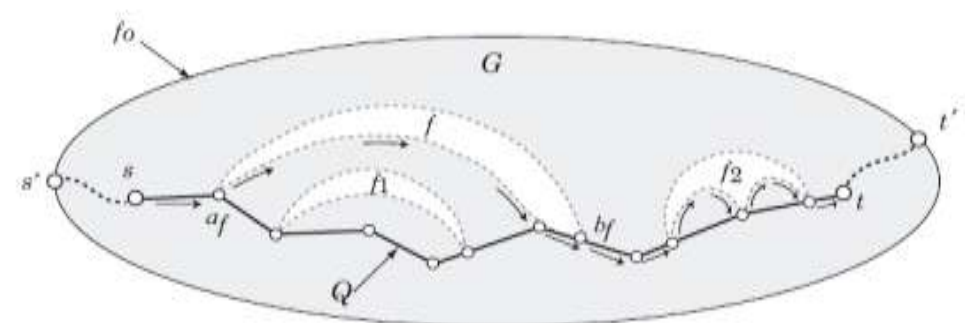

Figure 8. Construction of the left-aligned path $L(Q)$ from an inner path $Q$ between $s$ and $t$, where thick lines show $Q$ and the path following the arrows shows $L(Q)$.

Lemma 6 Let $G=(V, E, F)$ be an internally triconnected plane graph, and $Q$ be an inner path from a vertex $s$ to a vertex $t$. Then the left-aligned path $L(Q)$ is an inner path from $s$ to $t$, and no inner facial cycle arches $L(Q)$ on the left side. Moreover, if no inner facial cycle arches $Q$ on the right side, then $L(Q)$ is an archfree path.

Corollary 1 For any inner path $Q$ from s to $t$ in an internally triconnected plane graph $G$, the right-aligned path $R(L(Q))$ of the left-aligned path $L(Q)$ is an archfree path.

Lemma 7 For a triconnected plane graph $G=(V, E, F)$, let $S$ be a subset of $V\left(f_{o}\right)$ with $|S| \geq 2$. Then $G$ contains an archfree tree $T$ such that the set of leaves of $T$ is equal to $S$. Such a tree $T$ can be obtained in linear time.

\subsubsection{Convex Drawing with a Convex Boundary}

Lemma $8[3,4]$ Let $G=(V, E, F)$ be a biconnected plane graph. Then a drawing $\varphi$ of $f_{o}$ on a convex polygon $P$ can be extended to a convex drawing of $G$ if and only if the following conditions (i)-(iii) hold:

(i) For each inner vertex $v$ with $d_{G}(v) \geq 3$, there exist three paths disjoint except $v$, each connecting $v$ and an outer vertex;

(ii) Every cycle of $G$ which has no outer edge has at least three vertices $v$ with $d_{G}(v) \geq 3$;

(iii) Let $Q_{1}, Q_{2}, \ldots, Q_{k}$ be the subpaths of $f_{o}$, each corresponding to a side of $P$. The graph $G-V\left(f_{o}\right)$ has no component $H$ such that all the outer vertices adjacent to vertices in $H$ are contained in a single path $Q_{i}$, and there is no inner edge $(u, v)$ whose end vertices are contained in a single path $Q_{i}$.

Since every inner vertex of degree 2 must be drawn as a point sub-dividing a line segment in any convex drawing, we can assume without loss of generality that a given biconnected plane graph has no inner vertex of degree 2 . Then Lemma 8 can be restated as follows.

Lemma 9 Let $G=(V, E, F)$ be a biconnected plane graph which has no inner vertex with degree 2. Then a drawing $\varphi$ of $f_{o}$ on a convex polygon $P$ can be extended to a convex drawing of $G$ if and only if the following conditions (a) and (b) hold:

(a) $G$ is internally triconnected.

(b) Let $Q_{1}, Q_{2}, \ldots, Q_{k}$ be the subpaths of $f_{o}$, each corresponding to a side of $P$. Each $Q_{i}$ is an archfree path in $G$. 


\subsubsection{Convex Drawing with Star-Shaped Boundary Constraints}

A kernel $K(P)$ of a polygon $P$ is the set of all points from which all points in $P$ are visible. The boundary of a kernel, if any, is a convex polygon. A polygon $P$ is called star-shaped if $K(P) \neq \varnothing$. Throughout the paper, we assume that for a given star-shaped polygon, its kernel has a positive area.

Lemma 10 Let $G=(V, E, F)$ be a triconnected plane graph, and $\varphi$ be a drawing of the outer facial cycle $f_{o}$ on a star-shaped polygon P. For drawing $\varphi$, let $S$ be a valid subset of $V\left(f_{o}\right)$, and $T$ be an archfree tree whose leaf set is $S$. Then $\varphi$ can be extended to a strictly convex drawing $\psi$ of $H=T+f_{o}$. Such a drawing $\psi$ can be obtained in linear time.

The authors prove that a drawing $\varphi$ of $f o$ on $P$ can be extended to a strictly convex drawing $\psi$ of $H=T$ $+f_{o}$ such that $\psi$ satisfies the following two conditions:

(1) All non-leaf vertices of $T$ are drawn strictly inside $K$.

(2) The angle $\left(\psi\left(a_{u}\right), u, \psi\left(b_{u}\right)\right)<\pi$ for all fringe vertices $u$ except for a wide fringe vertex.

Case-1: $h \geq 3$. Let $T^{\prime}$ be the tree obtained from $T$ by removing leaves $v_{2}, v_{3}, \ldots, v_{h-1}, S^{\prime}=S-\left\{v_{2}, v_{3}, \ldots\right.$ . , $\left.v_{h-1}\right\}, f_{o}^{\prime}$ be the cycle obtained from $f_{o}$ by replacing its subpath from $v_{1}$ to $v_{h}$ with an edge $\left(v_{1}, v_{h}\right)$. Let $P^{\prime}$ be the polygon obtained from $P$ by replacing the edges between $v_{1}$ and $v_{h}$ with a single edge, and $\varphi$ ' be the resulting drawing of $f^{\prime}{ }_{o}$ on $P^{\prime}$ (see Fig. 9(b)). Note that $v *$ remains a non-wide fringe vertex in $T$ ' and hence, $S^{\prime}$ is valid in $P^{\prime}$. By the inductive hypothesis, $\varphi^{\prime}$ can be extended to a strictly convex drawing $\psi^{\prime}$ of $H^{\prime}=T^{\prime}+f^{\prime}$, that satisfies (1) and (2) (see Fig. 9(b)). We show that the original drawing $\varphi$ of $f_{o}$ on $P$ can be extended to a strictly convex drawing $\psi$ of $H=T+f_{o}$ satisfying (1) and (2).

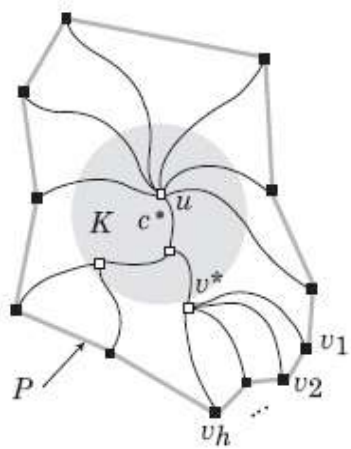

(a)

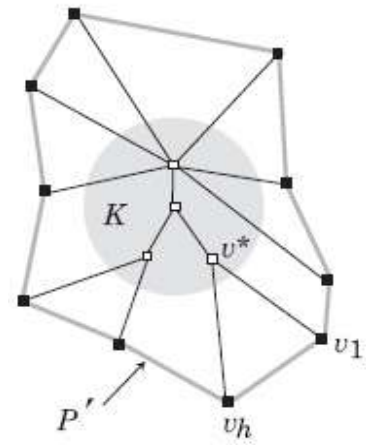

(b)

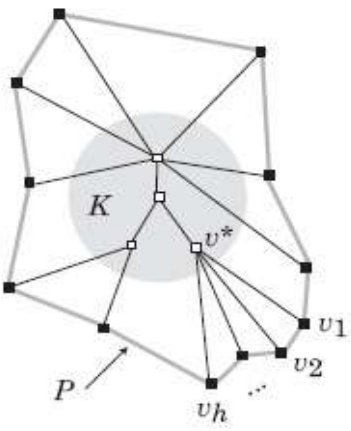

(c)

Figure 9. (a) Reducing the number of leaves adjacent to $v *$ in tree $T$, (b) Re-inserting the deleted leaves in a convex drawing of $H^{\prime}=T^{\prime}+f_{o}^{\prime}$ with boundary $P^{\prime}$, (c) A convex drawing of $H=T+f_{o}$ with boundary $P$.

Case-2: $h=2$. Let $w$ be the unique neighbor of $v *$ with $d_{T}(w) \geq 3$, and $e_{a}=\left(w, u_{a}\right)$ (respectively, $e_{b}=$ $\left.\left(w, u_{b}\right)\right)$ be the edge incident to $w$ that appears immediately before edge $(w, v *)$ (respectively, immediately after edge $(w, v *))$ when we walk around $w$ in the clockwise order. Since $d_{T}(w) \geq 3$, we have $e_{a} \neq e_{b}$ (see Fig. 10(a)).

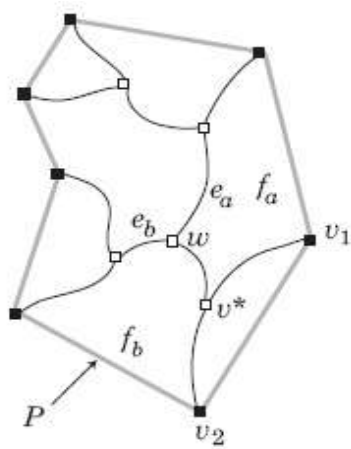

(a)

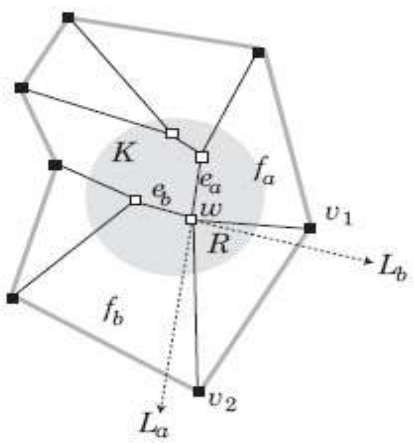

(b)

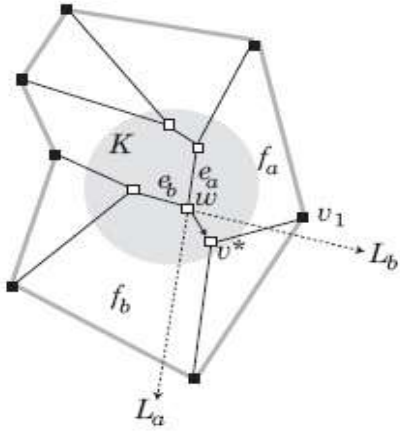

(c)

Figure 10. (a) Contracting edge $(v *, w)$ in tree $T$, (b) Re-inserting a contracted edge $(v *, w)$ in a convex drawing $\psi^{\prime}$ of $H^{\prime}=T^{\prime}+f_{o}$, (c) A convex drawing of $H=T+f_{o}$ with boundary $P$.

Theorem 3 Every drawing $\varphi$ of the outer facial cycle $f_{o}$ of a triconnected plane graph $G=(V, E, F)$ on a starshaped polygon can be extended to a convex drawing $\psi_{G}$ of $G$. Such a drawing $\psi_{G}$ can be computed in linear time. 


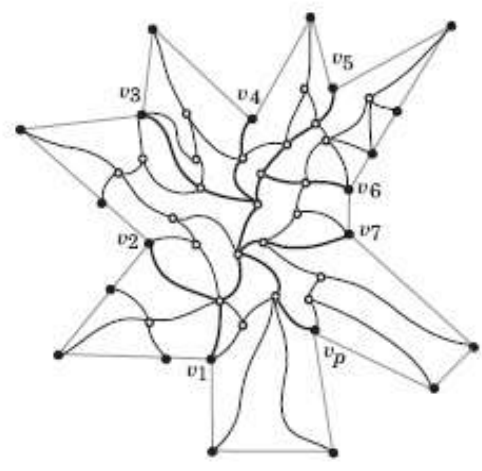

(a)

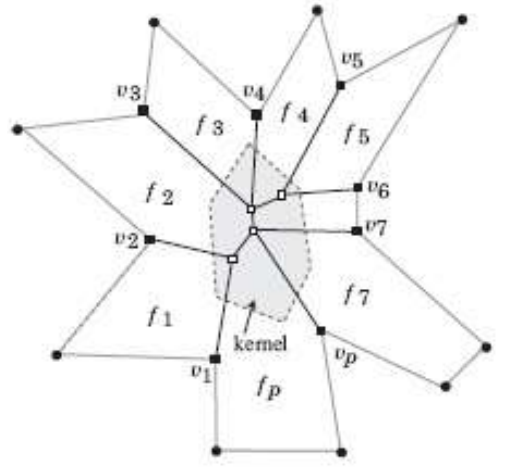

(b)

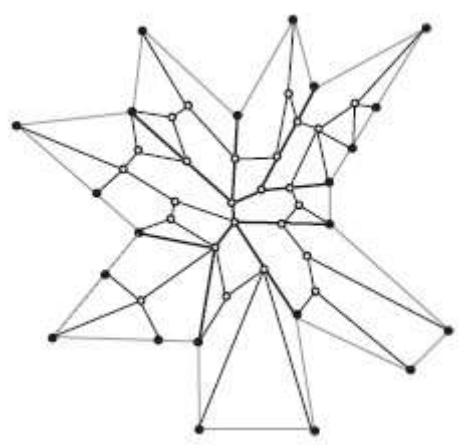

(c)

Figure 11. (a) A triconnected plane graph $G=(V, E, F)$ with a star-shaped boundary $P$, where an archfree tree $T$ is denoted by thick black lines, (b) A convex drawing of $H=T+f_{o}$ with boundary $P$, (c) A convex drawing of $G$ with boundary $P$.

\subsubsection{Convex Drawing with Crown-Shaped Boundary Constraints}

A polygon $P$ is called crown-shaped if it has a side $Q$ of $P$ and a point $p *$ outside $P$ such that, for any point $p$ inside $P$, the line segment $\left(p, p^{*}\right)$ does not intersect with any other side of $P$ than $Q$ (i.e., all points in $P$ are visible from a point $p *$ if $Q$ is removed from $P$ ). The authors call such a side $Q$ and a point $p *$ the base side and the pivot of a crown-shaped polygon. Note that any two points in the boundary of $P$ and $p *$ are not collinear. For example, Fig. 6(c) and Fig. 12(a) show plane graphs whose boundaries are drawn as crown-shaped polygons. The authors easily see that if we divide a star-shaped polygon into two or more polygons by a single straight line, then each of the resulting polygons is either star-shaped or crown-shaped.

As shown in Fig. 6(c), there is a triconnected plane graph with a crown-shaped boundary that cannot be extended to a convex drawing. The authors show that four-connectivity suffices for a plane graph with a crownshaped boundary to admit a convex drawing.

Theorem 4 Every drawing $\varphi$ of the outer facial cycle $f_{o}$ of a four-connected plane graph $G=(V, E, F)$ on a crown-shaped polygon can be extended to a convex drawing $\psi_{G}$ of $G$. Such a drawing $\psi_{G}$ can be computed in linear time.

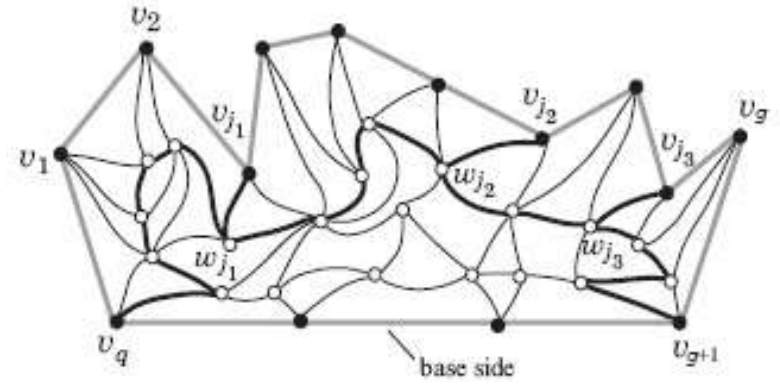

(a)

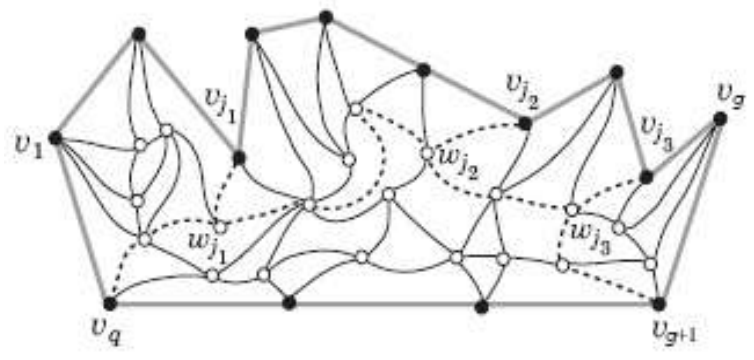

(b)

Figure 12. (a) A four-connected plane graph $G=(V, E, F)$ with a crown-shaped boundary $P$, where the path $Q$, from $v_{q}$ to $v_{g}$ of the boundary of $G$ ' and matching $M$ are displayed as thick lines, (b) A archfree tree $T *$ obtained from tree $T$ with $E(T)=E\left(Q^{\prime}\right) \cup M$, where edges in $T$ are displayed as dashed lines.

Lemma 11 Let $G=(V, E, F)$ be a four-connected plane graph, and $\varphi$ be a drawing of the outer facial cycle $f_{o}$ on a crown-shaped polygon $P$. Let $v_{g+1}$ and $v_{q}$ denote the vertices drawn as the endvertices of the base side $Q$ of $P$, and $V * \neq \varnothing$ be the set of vertices $v \in V\left(f_{o}\right)$ such that $\varphi(v)$ is a concave apex of $P$. Let $T *$ be an archfree tree such that its leaf set is $V * U\left\{v_{g+1}, v_{q}\right\}$ and its edge set can be partitioned into a path between $v_{g+1}$ and $v_{q}$ and a matching covering $V *$. Then $\varphi$ can be extended to a strictly convex drawing $\psi$ of $H=T *+f_{o}$. Such a drawing $\psi$ can be obtained in linear time.

In this paper [11], the authors initiate a new problem of convex drawings of planar graphs with nonconvex boundary constraints. It is proved that every triconnected plane graph with a star-shaped boundary admits an inner-convex drawing. We present a linear time algorithm for computing such an inner-convex drawing. Similar results hold for four-connected graphs with crown-shaped polygon. This algorithm runs in linear time. 


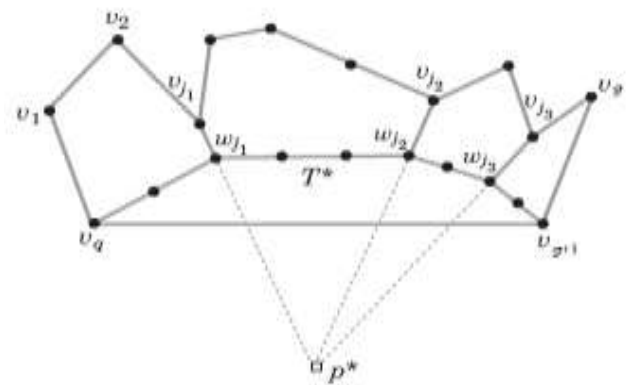

(a)

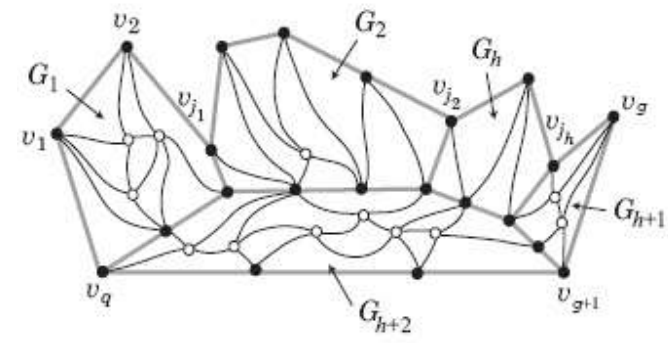

(b)

Figure 13. (a) A strictly convex drawing $\psi$ of $H=T *+f_{o}$, (b) Internally triconnected plane graphs $G_{i}, i=1,2, \ldots$ . , $h+2$, with convex boundaries.

\subsection{Convex Drawings of Hierarchical Planar Graphs}

In 2010, Hong and Nagamochi [13] proved that every internally triconnected hierarchical plane graph with the outer facial cycle drawn as a convex polygon admits a convex drawing.

Hierarchical graphs are graphs with layering structures. Hierarchical graphs (sometimes called level graphs) are directed graphs with vertices assigned into layers (or levels). These graphs are drawn with vertices of a layer on the same horizontal line, and edges as curves monotonic in $y$ direction. An edge with a tail $u$ and a head $v$ is denoted by $(u, v)$. For each vertex $\mathrm{v}$ in $\mathrm{H}$, denote $\{u \in V \mid(v, u) \in A\}$ by $\mathrm{V}^{+}{ }_{\mathrm{H}}(\mathrm{v})$ and $\{u \in V \mid(u, v) \in$ $A\}$ by $\mathrm{V}_{\mathrm{H}}^{-}(\mathrm{v})$. A vertex $\mathrm{v}$ is called a source, if $V_{H}(v)=\varnothing$ and $\mathrm{v}$ is called a sink, if $V_{H}^{+}(v)=\varnothing$. For a non-sink vertex $\mathrm{v}$, a vertex $\mathrm{w} \in \mathrm{V}_{\mathrm{H}}^{+}(\mathrm{v})$ is called an up-neighbor of $v$ (see Fig. 14), and $\mathrm{w}$ is called the highest upneighbor, if $\lambda(w)=\max \left\{\lambda(u) \mid u \in V_{H}^{+}(v)\right\}$. For a non-source vertex $v$, a vertex $\mathrm{w} \in \mathrm{V}_{\mathrm{H}}^{-}(\mathrm{v})$ is called a downneighbor of $v$, and $w$ is called the lowest down-neighbor, if $\lambda(w)=\min \left\{\lambda(u) \mid u \in V_{H}(v)\right\}$. A hierarchical graph is hierarchical planar (h-planar) (or level-planar) if it admits a drawing without edge crossings. A plane embedded hierarchical graph a hierarchical plane graph. If a hierarchical plane graph has only one source $s$ and one sink $t$, then we call it a hierarchical-st plane graph. Hierarchical-st plane graph is a connected graph, and its source $s$ and sink $t$ must lie on the bottom layer and the top layer, respectively. The head $w$ of the rightmost (respectively leftmost) edge outgoing from $v$ is called the right up-neighbor (respectively the left up-neighbor) of $v$, and is denoted by $r_{H}^{+}(v)$ (respectively $\ell_{\mathrm{H}}^{+}(\mathrm{v})$ ). The right down-neighbor $\mathrm{r}_{\mathrm{H}}^{-}(\mathrm{v})$ and the left down-neighbor $\ell_{\mathrm{H}}^{-}(\mathrm{v})$ of $\mathrm{v}$ are defined analogously.

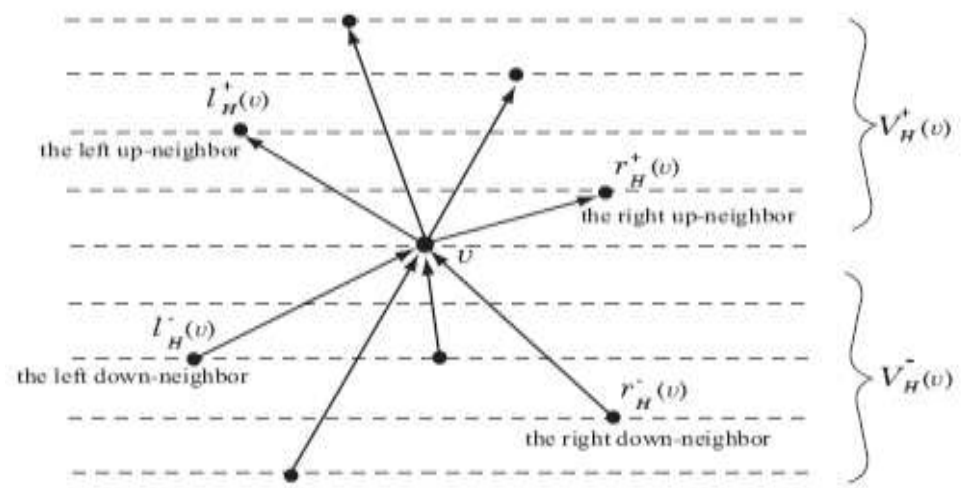

Figure 14. Left-right relations in $\mathrm{V}_{\mathrm{H}}^{-}(\mathrm{v})$ and $\mathrm{V}_{\mathrm{H}}^{+}(\mathrm{v})$.

The following theorem implies that every hierarchical-st plane graph $H$ admits a straight-line hierarchical planar drawing.

Theorem 5 [15] Let $H$ be a triangulated hierarchical-st plane graph, and its outer facial cycle $f_{o}$ be drawn as a convex polygon $P$ such that, for each chord $(u, z)$ of $f_{o}$, on each of the two paths of cycle $C$ between $u$ and $z$, there exists a vertex $v$ which is drawn as a convex apex of polygon $P$. Then there exists a planar straight-line hierarchical drawing of $H$ with external face $P$, and such a drawing can be constructed in linear time.

Lemma 11 Let $H$ be a hierarchical-st plane graph that satisfies conditions (a) and (b) in Lemma 9 [11]. For any monotonic inner path $Q$ from a vertex $u$ to a vertex $v, R(L(Q))$ is a monotonic archfree path.

Proof: By Corollary 1 [11], $R(L(Q))$ is an archfree path. It suffices to show that operation $L$ of constructing path $L(Q)$ from a monotonic inner path $Q$ preserves the monotonicity of $Q$. For this, we consider an 
inner facial cycle $f$ on the left side of $Q$, where $a_{f}$ and $b_{f}$ are the first and last vertices in $V(f) \cap V(Q)$ when we walk along path $Q$ from $s$ to $t$ (see Fig. 8), and prove that the subpath $P_{f}$ from $a_{f}$ to $b_{f}$ obtained by traversing $f$ in an anticlockwise direction is monotonic. If $P_{f}$ is not monotonic, then there are three vertices $u_{1}, u_{2}$ and $u_{3}$ which appear in this order on $P_{f}$ and whose $y$-coordinates $y\left(u_{1}\right), y\left(u_{2}\right)$ and $y\left(u_{3}\right)$ satisfice $y\left(u_{1}\right)>y\left(u_{2}\right)<y\left(u_{3}\right)$. Implies that $u_{2}$ is another source, since $H$ is a hierarchical-st plane graph, which contradicts the situation where $H$ has no other source than $s$. This proves the lemma. In this paper, the authors prove the following result.

Theorem 6 For every hierarchical-st plane graph $H$ which is internally triconnected, any convex polygon $P$ for the outer facial cycle $f_{o}$ can be extended to a convex drawing of $H$. Such a drawing can be computed in $O\left(n^{2}\right)$ time.

Proof: Prove by induction on the number of inner faces. No inner vertex of degree 2. The theorem holds if $H$ has only one inner face. Consider an internally triconnected hierarchical-st plane graph $H$, and assume that the theorem holds for any triconnected hierarchical-st plane graph which has a smaller number of inner faces than $H$. There are two Cases:

Case 1: There is an outer vertex $v(\neq s, t)$ of degree 2 .

Case 2: Every outer vertex $v(\neq s, t)$ is of at least 3 degrees

Case 1: Let $v$ and $v^{\prime \prime}$ be the up- and down-neighbours of $v$. If $v$ is a flat apex in $P$, then we see that $H$ with $P$ has a convex drawing $D$ since replacing two edges $\left(v^{\prime \prime}, v\right)$ and $\left(v, v^{\prime}\right)$ with a new edge $\left(v^{\prime \prime}, v^{\prime}\right)$ deleting vertex $\mathrm{v}$ results in a hierarchical-st plane graph $H$ with a new convex boundary $P$, which admits a convex drawing $D$ by the induction hypothesis. Now assume that $v$ is a convex apex in $P$. Construct a hierarchical-st plane graph $H$ from $H$ by removing $v$ and adding a new edge $\left(v^{\prime \prime}, v^{\prime}\right)$ if $v^{\prime \prime} \notin V_{H}\left(v^{\prime}\right)$. Let $P$ be the polygon obtained by replacing the segments $\left(v^{\prime \prime}, v\right)$ and $\left(v, v^{\prime}\right)$ in $P$ with a new segment $\left(\mathrm{v}^{\prime \prime}, \mathrm{v}^{\prime}\right)$, which will form a new side of $P$. H with boundary $P$ satisfies conditions (a) and (b) in Lemma 9 [11].

Case 2: Consider the leftmost monotonic path $Q_{v}$ from $v$ and let w be the first vertex in $Q_{v}$ that has at least one down-neighbor. $Q_{1}$ be the subpath from $v$ to $w$ along $Q_{v} . Q_{1}$ is a subpath of an inner facial cycle $f_{v}$ which contains $v$, and is an archfree path. Consider the two subcases:

(i) $\quad w$ is an outer vertex (see Fig. 15(a)). The outer facial cycle $f_{o}$ together with path $Q_{l}$ splits the interior of $P$ into two regions, say $R_{l}$ and $R_{2}$. We split $H$ into two hierarchical-st plane graphs $H_{l}$ and $H_{2}$ such that $H_{l}$ and $H_{2}$ share $Q_{1}$.

(ii) $\quad w$ is an inner vertex (see Fig. 15(b)). Choose a monotonic path $Q_{z}$ that starts from $w$ and ends up with an outer vertex $z$. Also choose a monotonic path $Q_{u}$ to $w$, from an outer vertex $u$.

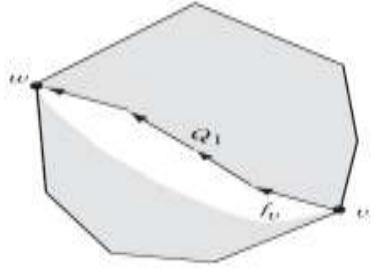

(a)

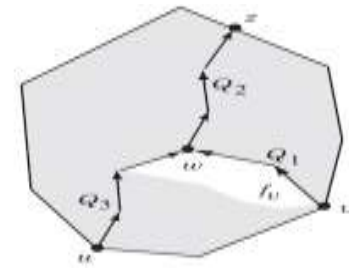

(b)

Figure 15. (a) A monotonic archfree path between two outer vertices $v$ and $w$. (b) Constructing three monotonic archfree paths starting from $v, w$ and $u$, respectively.

By Lemma 11, $\mathrm{H}$ has monotonic archfree paths $Q_{2}$ from $w$ to $z$ and $Q_{3}$ from $u$ to $w$. By placing $w$ at a point in the interior of $P$, we draw each path $Q_{i}, i=1,2,3$ as a straight line between its end points, by which the $x$ coordinates of all points on $Q_{i}$ are uniquely determined.

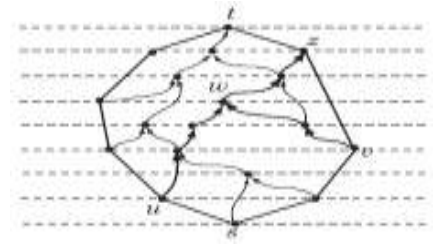

(a)

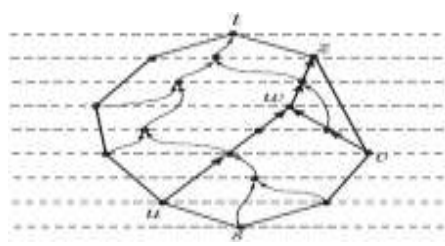

(b)

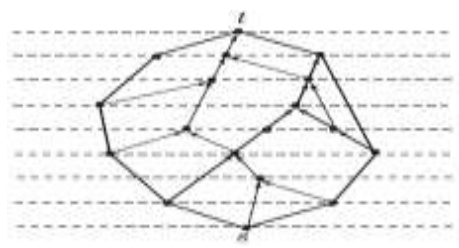

(c)

Figure 16. (a) A hierarchical-st plane graph $H$ with a convex polygon $P$, where three monotonic paths with thick lines are archfree paths, (b) Three hierarchical-st plane graphs obtained by the archfree paths, (c) A convex drawing of $H$ in (a).It is proved that every internally triconnected hierarchical plane graph with the outer facialcycle drawn as a convex polygon admits a convex drawing in $\mathrm{O}\left(\mathrm{n}^{2}\right)$ time. 


\section{CONCLUSION}

Different types of algorithm are given in different time for convex drawing of planar graphs. Different variations are considered. This paper describes few of them. Still now researchers work on this.

Recently in 2010 Hong and Nagamochi [14] present a linear time algorithm for symmetric convex drawings of planar graphs. In this paper, they prove that given an internally triconnected plane graph with symmetries, there exists a convex drawing of the graph which displays the given symmetries. The authors present a linear-time algorithm for constructing symmetric convex drawings of internally triconnected planar graphs (see Fig.17).
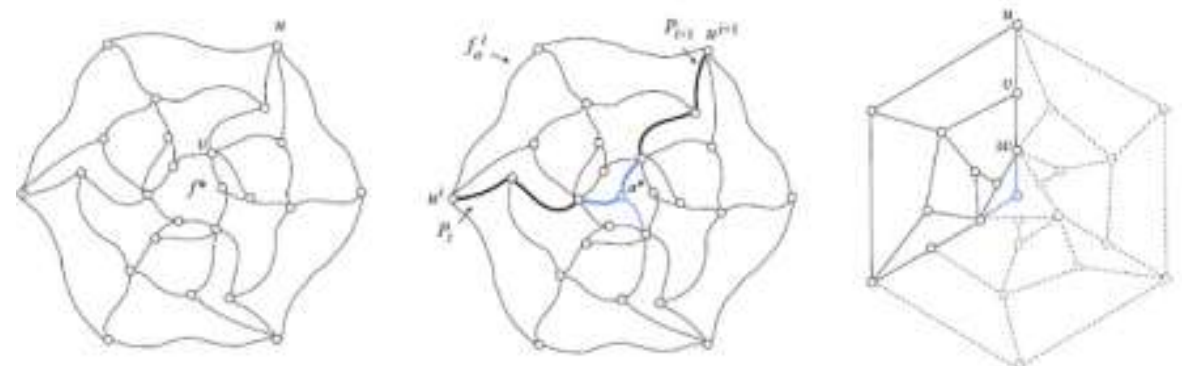

Figure 17. Symmetric convex drawings of planar graph.

In $2011 \mathrm{He}$ and Zhang present [16] on succinct convex greedy drawing of 3-connected plane graphs. Recently in 2012 again Hong and Nagamochi present [17] a linear-time algorithm for star-shaped drawings of planar graphs with the minimum number of concave corners.

It would be interesting to apply the notion of archfree paths/trees to convex drawings or inner convex drawings of other types of graphs. Further, adding more constraints such as angles of vertices to convex representations may be an interesting research direction in the future.

\section{REFERENCES}

[1] W. T. Tutte, Convex representations of graphs, Proc. of London Math, Soc. 10, no. 3, 1960, 304-320.

[2] W. T. Tuttle, How to draw a graph, Proc. London math, Soc. 13, 1963, 743-768.

[3] C. Thomassen, Plane representations of graphs, Progress in Graph Theory, J. A. Bondy and U. S. R. Murty (Eds.), Academic Press, 1984, 43-69.

[4] N. Chiba, T. Yamanouchi and T. Nishizeki, Linear algorithms for convex drawings of planar Graphs, Progress in Graph Theory, Academic Press, 1984, 153-173.

[5] N. Bonichon, S. Felsner and M. Mosbah, Convex drawings of 3-connected plane graphs, Proc. of Graph Drawing 2004, 2005, 6070 .

[6] M. Chrobak, M. T. Goodrich and R. Tamassia, Convex drawings of graphs in two and three Dimensions, Proc. of SoCG 1996, 1996, 319-328.

[7] M. Chrobak and G. Kant, Convex grid drawings of 3-connected planar graphs, International Journal of Computational Geometry and Applications, 7, 1997, 211-223.

[8] K. Miura, S. Nakano and T. Nishizeki, Convex grid drawings of four-connected plane Graphs, International Journal of Foundations of Computer Science, 17(5), 2006, 1031-1060.

[9] K. Miura, M. Azuma and T. Nishizeki, Convex drawings of plane graphs of minimum outer apices, Proc. of Graph Drawing 2005, 2006, 297-308.

[10] G. Rote, Strictly convex drawings of planar graphs, Proc. of SODA 2005, 2005, 728-734.

[11] SH. Hong and H. Nagamochi, Convex drawings of graphs with non-convex boundary, Proc. of WG 2006, 2006, 113-124

[12] G Di Battista, F Frati, M Patrignani, Non-convex representations of graphs, Graph Drawing, Springer, 2009.

[13] SH. Hong and H. Nagamochi, Convex drawings of hierarchical planar graphs and clustered planar graphs, Journal of Discrete Algorithms, Elsevier, 2010.

[14] SH. Hong and H. Nagamochi, Linear time algorithm for symmetric convex drawings of planar graphs, Algorithmica, Springer, 2010.

[15] P. Eades, Q. Feng, X. Lin and H. Nagamochi, Straight-line drawing algorithms for hierarchical graphs and clustered graphs, Algorithmica, 44, 2006, 1-32.

[16] X He, H Zhang, On succinct convex greedy drawing of 3-connected plane graphs, Proceedings of the Twenty-Second Annual ACMSIAM, 2011

[17] SH Hong, H Nagamochi, A linear-time algorithm for star-shaped drawings of planar graphs with the minimum number of concave corners, Algorithmica, Springer, 2012. 